\title{
Supramolecular arrangements in the crystal structures and the interaction energy calculations of resonance-assisted hydrogen-bridged (RAHB) rings - RAHB/RAHB and RAHB/C 6 -aromatic contacts
}

\author{
J. P. Blagojević Filipović ${ }^{1}$, S. D. Zarić \\ ${ }^{1}$ Innovation Centre of the Faculty of Chemistry, Studentski trg 12-16, Belgrade, Serbia, \\ ${ }^{2}$ Faculty of Chemistry, University of Belgrade, Studentski trg 12-16, Belgrade, Serbia \\ szaric@chem.bg.ac.rs
}

The Cambridge Structural Database (CSD) is searched for mutual contacts between six-membered resonance-assisted hydrogenbridged rings (RAHB) (the example of a fragment is shown in Fig. 1a) [1] and for contacts between six-membered RAHB rings and $\mathrm{C}_{6}$-aromatic rings (Fig. 1b). There is a quite large prevalence of parallel contacts in the set of RAHB/RAHB contacts, since $91 \%$ from totally 678 contacts found are parallel contacts, mostly with antiparallel orientation of the rings [1]. At the other side, the prevalence of parallel contacts in the set of RAHB/ $\mathrm{C}_{6}$-aromatic contacts is not so pronounced, since $59 \%$ from totally 677 contacts found are parallel contacts. The distances between the interacting ring planes are mostly between 3.0 and $4.0 \AA$, while horizontal displacements are mostly in the range $0.0-3.0 \AA$ in both parallel RAHB/RAHB and $\mathrm{RAHB} / \mathrm{C}_{6}$-aromatic contacts.

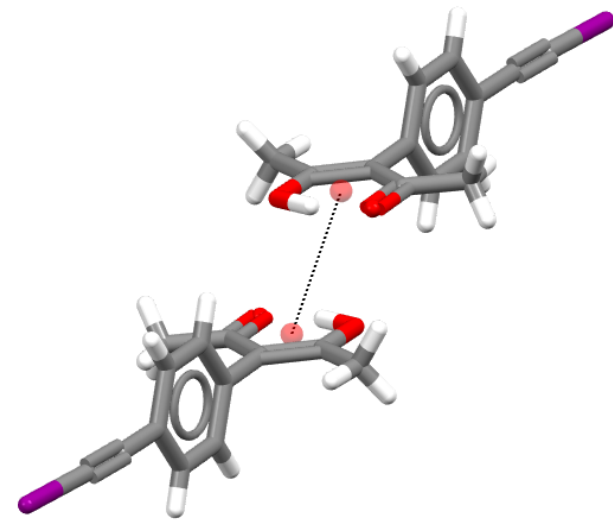

a)

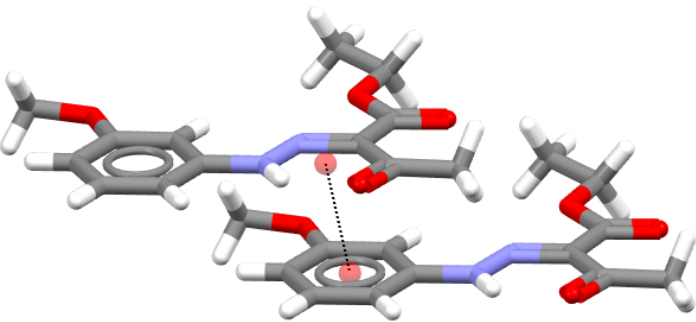

b)

Figure 1. The examples of fragments used in CSD search for a) six-membered RAHB/RAHB contacts; b) six-membered RAHB/C $6^{-}$ aromatic contacts

The interaction energy calculations were performed on stacked dimer model systems based on abundance in the CSD. The strongest calculated RAHB/RAHB interaction is $-4.7 \mathrm{kcal} / \mathrm{mol}$, while the strongest calculated RAHB/benzene interaction is significantly weaker $-3.7 \mathrm{kcal} / \mathrm{mol}$. However, RAHB/RAHB stacking interactions can be stronger or weaker than the corresponding RAHB/benzene stacking interactions, depending on the RAHB ring system. The Symmetry Adopted Perturbation Theory (SAPT) calculations show that the dominant contribution in total RAHB/RAHB stacking interaction energy is the dispersion term, which can be mostly or completely cancelled by the exchange repulsion term, hence, the electrostatic term can be effectively dominant. Depending on the RAHB ring system, the electrostatic contribution can be practically equal to the net dispersion contribution (the sum of dispersion and exchange-repulsion terms) [1]. The electrostatic term is effectively dominant in all RAHB/benzene systems observed, due to the almost complete cancellation of the dispersion by the exchange-repulsion terms.

[1] Blagojević Filipović, J. P., Hall, M. B. \& Zarić, S. D. (2019).Cryst. Growth Des. 19, 5619.

Keywords: stacking; RAHB; CSD

This work was supported by the Serbian Ministry of Education, Science and Technological Development (Grant number 172065). 